\title{
Editorial
}

\section{Animal Models-Decoding the Molecular Biology of Oral Cancer}

Animal models have long been used to understand the initiation and progression of carcinogenesis, including that of oral mucosa. ${ }^{1}$ One of the earliest models used was the chemical-induced oral cancer model, among which the Syrian Hamster check pouch was preferred for its ideal anatomical location and physiological features. ${ }^{2}$ Salley et al ${ }^{3}$ demonstrated that the cheek pouch mucosa underwent gradual changes from hyperplasia, carcinoma in situ to squamous cell carcinoma when exposed to polycyclic hydrocarbon 9, 10 dimethyl-1,2, benzanthracene (DMBA). Morris ${ }^{4}$ standardized the dosage of carcinogen to $0.5 \%$ solution of DMBA in acetone and established that 5-week old animals were ideal to induce tumor with minimum time lag and maximum yield. Lin et $\mathrm{al}^{5}$ demonstrated the synergistic effect of arecaidine with DMBA. The areca nut in itself was not capable of inducing carcinogenesis but acts as a promoter and halves the time duration necessary for DMBA-induced mutagenesis. The rat oral cavity model was established as an alternative to the cheek pouch model. Both the models were successful in demonstrating the alterations in the expression of oncogenes and proliferative markers and provided a basic understanding of the role of neovascularization in tumor progression. The major disadvantage of the cancer animal model is labor intensity and prolonged handling time. There was also a considerable concern over the fact that there was a histological difference between the hamster and human oral mucosa.

Transplantable tumors, transgenic animals and cocarcinogenicity models are some of the recent models finding approval in oral cancer research. In the transplanted tumor model, the tumor cells are placed on to animal to study its progression. The initial models consisted of severe combined immunedeficient (SCID) mice and nu/nu mice. Though immunosupression might aid in acceptance of the tumor, the host immunological response could not be assessed. Due to the lack of an appropriate immune response, it is near impossible to formulate a treatment modulation. The answer for the immune deficiency came in the form of syngeneic hosts. However the higher risk of bacterial contamination during resection, weak cellular activity made it extremely difficult in establishing the cancer cell line for the same. In addition, the primary tumors have lower sustainability in comparison to metastatic tumors. But, utilizing metastatic tumors produced a less differentiated and less organized cell line. Odukoya et al ${ }^{6}$ established an epidermoid carcinoma cell line (HCPC I) by applying DMBA in heavy mineral oil on the hamster cheek pouch model. They reported a doubling time of about 12 hours. The hamster cheek pouch model is considered as the ideal model even today due to its high tolerance to cancer cells. The absence of tumor rejection by the cheek pouch model is due to the presence of negligible amount of transplantation antigen-encoded genetic loci. Certain major disadvantages persist in this model including the need to inject a large dosage of tumor cells to sustain its proliferation. This limitation was overcome by the use of spontaneous models. $\mathrm{O}^{\prime}$ Malley et $\mathrm{al}^{7} \mathrm{demonstrated} \mathrm{that}$ when an immunocompetent $\mathrm{C} 3 \mathrm{H} / \mathrm{HeJ}$ mice was injected with SCC VII line into the floor of the mouth, the cancer line sustained and progressed to form local invasion plus regional and long-distance metastasis.

The transgenic and the knockout mice use tissue or cell-specific promoters driving tumor-specific oncogenes. They provide us a host with immunological interactions as close to human cancer as possible. There was a consistent difference in the progression of the tumors depending on the anatomical location. Tongue showed a higher proliferation and progression in comparison to the cheek mucosa. Cocarcinogenesis aids us in understanding the complex interactions between the carcinogens and the host's genetic make up. ${ }^{1}$ In the cocarcinogenesis models, the carcinogen and the artificially induced event including stimulating a chronic traumatic ulcer-induced carcinogenesis at a faster rate with significantly higher yield in comparison to administering the carcinogen alone. Regional and distant metastasis was not a consistent feature of cocarcinogenesis animal model which is in contrary is a significant cause for mortality in human cancer. To address this issue metastatic and angiogenetic models were introduced. ${ }^{1}$ Even the advanced transplanted animal models has a common disadvantage in that these cancer models were transplanted in the form of a mass of tumor cell or a larger organized tumor, none of which could stimulate the properties of cancer originating from a single cell. Though multiple animal models demonstrating tumor progression are flooding the research pool, a model representing the initial stages of oral cancer is still awaited. Developing an ideal model simulating the various stages of oral cancer may provide us with an understanding of its complex biology and aid us in creating early diagnostic markers and novel therapeutic targets. 


\section{REFERENCES}

1. Mognetti B, Carlo FD, Berta GN. Animal models in oral cancer research. Oral Oncology 2006;42:448-460.

2. Keyes PH, Dale PP. A preliminary survey of the pouches and dentition of the Syrian hamster. J Dent Res 1944;23:427-438.

3. Salley JJ. Experimental carcinogenesis in the cheek pouch of the Syrian hamster. J Dent Res 1954;33:253-262

4. Morris AL. Factors influencing experimental carcinogenesis in the hamster cheek pouch. J Dent Res 1961;40:3-15.

5. Lin LM, Chen YK, Lai DR, Huang YL. Minimal arecaidine concentrations showing a promotion effect during DMBA induced hamster cheek pouch carcinogenesis. J Oral Pathol Med 1996;25:65-68.

6. Odukoya O, Schwartz J, Weichselbaum R, Shklar G. An epidermoid carcinoma celline derived from hamster 7,12dimethylbenzaanthracene-induced buccal pouch tumors. J Natl Cancer Inst 1983;71:1253-1264.

7. O'Malley Jr BW, Cope KA, Johnson CS, Schwartz MR. A new immunocompetent murine model for oral cancer. Arch Otolaryngol Head Neck Surg 1997;123:20-24.

Shankargouda Patil

Associate Professor

Department of Oral Pathology and Microbiology

Faculty of Dental Sciences,

MS Ramaiah University of Applied Sciences

Bengaluru, Karnataka, India

Roopa Rao

Professor and Head

Department of Oral Pathology

Faculty of Dental Sciences

MS Ramaiah University of Applied Sciences

Bengaluru, Karnataka, India

Thirumal Raj

Final Year Postgraduate Student

Department of Oral Pathology

Faculty of Dental Sciences

M S Ramaiah University of Applied Sciences

Bengaluru, Karnataka, India 